\title{
Dusty plasma (Yukawa) rings
}

\author{
T. E. Sheridan* and J. C. Gallagher \\ Department of Physics and Astronomy, \\ Ohio Northern University, Ada, Ohio 45810 U.S.A.
}

\begin{abstract}
One-dimensional and quasi-one-dimensional strongly-coupled dusty plasma rings have been created experimentally. Longitudinal (acoustic) and transverse (optical) dispersion relations for the 1-ring were measured and found to be in very good agreement with the theory for an unbounded straight chain of particles interacting through a Yukawa (i.e., screened Coulomb or Debye-Hückel) potential. These rings provide a new system in which to study one-dimensional and quasi-onedimensional physics.
\end{abstract}

*Electronic address: t-sheridan@onu.edu 
One-dimensional (1D) chains of particles are an important model system for studying linear and nonlinear phenomena such as waves, instabilities, and diffusion [1-3]. A standard model is that of an unbounded straight chain of particles interacting through a given force. This system may represent a lumped model of a continuous system, such as a string, or may model the atomic lattice. Although infinite systems cannot be realized in practice, they can be approximated using large systems with periodic boundary conditions so that end effects are eliminated. One experimental system where it is possible to study strongly-coupled 1D chains is dusty (complex) plasma.

Dusty plasma is a system of microscopic material particles immersed in an electron-ion plasma. For typical laboratory conditions, the dust particles acquire a net charge $q<0$ and can be confined in a single-layer suspension above a horizontal electrode. Vertical confinement is due to the balance between the downward gravitational force and the average upward electrostatic force inside the plasma sheath. Horizontal confinement is often due to depressions in the plasma potential created by secondary electrodes. The interaction between dust particles is screened by the response of the electrons and ions so that the potential a distance $r$ from a dust particle is given by a Yukawa (screened Coulomb or Debye-Hückel) potential [4, 5]

$$
V(r)=\frac{1}{4 \pi \epsilon_{0}} \frac{q}{r} e^{-r / \lambda_{D}}
$$

where $\lambda_{D}$ is the Debye screening length. In principle, the interaction length can be varied from long to short range allowing study of both "plasma" and "condensed matter" regimes [6]. Since the dominant friction force on dust particles is neutral gas (Epstein) drag, wave modes may be underdamped at low gas pressures.

Previous experimental work on 1D dusty plasmas has focused on straight chains confined in highly anisotropic two-dimensional (2D) biharmonic wells [7-12]. Such systems have several drawbacks. First, the lattice constant $a$ is not a constant and increases at the ends of the chain. Second, the boundary conditions are not periodic, so that comparisons to unbounded theories are problematic. Third, it is difficult to make very long straight chains since 1D chains are unstable against the zigzag when the lattice constant is below a critical value [7, 12]. To overcome these drawbacks it may be possible to create a one-dimensional Coulomb ring [13] or dusty plasma ring [14] using a two-dimensional (2D) annular potential well. A dusty plasma ring will provide the most direct test of the theory for lattice waves in unbounded Yukawa chains [15], as well as a system with which to explore phenomena such 
as single-file diffusion [16] and the properties of quasi-one-dimensional systems [17].

In the present work we demonstrate that it is possible to create one-dimensional and quasi-one-dimensional dusty plasma rings in experiment, thereby providing a real system that models a chain of interacting particles with periodic boundary conditions. We measure the longitudinal and transverse dispersion relations for a 1-ring using thermal fluctuations, and confirm that the waves are well described by the theory of an unbounded 1D Yukawa chain.

We briefly review dusty plasma ring theory [14]. The dusty plasma consists of $n$ identical particles with mass $m$ and charge $q$ at positions $\left\{x_{i}, y_{i}\right\}$ confined in a 2D annular potential well [13. The potential energy of an isolated particle in this well is

$$
U_{\text {well }}(r)=\frac{1}{2} m \omega_{0}^{2}(r-s)^{2},
$$

where $\omega_{0}$ is the single-particle radial oscillation frequency, $r$ is a radial coordinate and $s$ is the radius at which the well potential energy is a minimum. The total potential energy of a particle configuration is

$$
U=\frac{1}{2} m \omega_{0}^{2} \sum_{i=1}^{n}\left(\sqrt{x_{i}^{2}+y_{i}^{2}}-s\right)^{2}+\frac{q^{2}}{4 \pi \epsilon_{0}} \sum_{i<j}^{n} \frac{e^{-r_{i j} / \lambda_{D}}}{r_{i j}}
$$

where the second sum is the interaction potential energy for all unique pairs of particles and $r_{i j}$ is the distance between particles $i$ and $j$. The potential energy $U$ is nondimensionalized by defining variables

$$
\kappa=\frac{r_{0}}{\lambda_{D}}, \sigma=\frac{s}{r_{0}}, \xi_{i}=\frac{x_{i}}{r_{0}}, \eta_{i}=\frac{y_{i}}{r_{0}}, \rho_{i j}=\frac{r_{i j}}{r_{0}},
$$

where the characteristic length and energy are

$$
r_{0}^{3}=\frac{1}{\frac{1}{2} m \omega_{0}^{2}} \frac{q^{2}}{4 \pi \epsilon_{0}}, U_{0}^{3}=\frac{1}{2} m \omega_{0}^{2}\left(\frac{q^{2}}{4 \pi \epsilon_{0}}\right)^{2},
$$

to give

$$
\frac{U}{U_{0}}=\sum_{i=1}^{n}\left(\sqrt{\xi_{i}^{2}+\eta_{i}^{2}}-\sigma\right)^{2}+\sum_{i<j}^{n} \frac{e^{-\kappa \rho_{i j}}}{\rho_{i j}} .
$$

In dimensionless form, time-independent configurations $\left\{\xi_{i}, \eta_{i}\right\}$ are determined by three parameters: $n, \kappa$ and $\sigma$. Here $\kappa$ is the Debye shielding parameter and $\sigma$ is the radius at which the confining well has its minimum. This model was shown [14] to have onedimensional ring solutions (i.e., 1-rings). A 1-ring is unstable against the zigzag instability 
when the particle density exceeds a critical value, which may lead to the creation of quasi-1D systems consisting of concentric rings, e.g., 2-rings.

For a 1D chain with lattice parameter $a$ the dimensionless lattice parameter is $\alpha=a / r_{0}$. The curvature of the ring is negligible when $s \gg a$ and $s \gg \lambda_{D}$. In this case, an appropriate shielding parameter is

$$
\bar{\kappa}=\frac{a}{\lambda_{D}}=\alpha \kappa .
$$

The dispersion relation for an undamped longitudinal (acoustic) wave in an unbounded straight 1D Yukawa chain is [15]

$$
\frac{\omega_{l}^{2}}{\omega_{0}^{2}}=\frac{4}{\alpha^{3}} \sum_{j=1}^{2} \frac{e^{-j \bar{\kappa}}}{j^{3}}\left[1+j \bar{\kappa}+\frac{1}{2}(j \bar{\kappa})^{2}\right] \sin ^{2}\left(\frac{j k a}{2}\right),
$$

where $\omega_{l}$ is the longitudinal wave frequency and $k$ is the real wavenumber. The dispersion for the transverse (optical) mode is [10]

$$
\frac{\omega_{t}^{2}}{\omega_{0}^{2}}=1-\frac{1}{\alpha^{3}} \sum_{j=1}^{\infty} \frac{e^{-j \bar{\kappa}}}{j^{3}}(1+j \bar{\kappa})[1-\cos (j k a)],
$$

where $\omega_{t}$ is the transverse wave frequency. The optical mode is a backward wave. These dispersion relations were previously found to be in good agreement with theoretical normal mode spectra for a dusty plasma 1-ring [14].

Our experimental setup is shown in Fig. 1. Monodisperse microscopic $(8.94 \pm 0.09 \mu \mathrm{m}$ diameter) melamine dust particles are levitated in an rf plasma discharge above the capacitively-coupled powered electrode in the DONUT (Dusty ONU experimenT) apparatus [7, 18 21]. Dust confinement is due to a 2D annular potential well created by cutting an annular rectangular groove in the surface of the $89-\mathrm{mm}$ powered electrode. The groove has an inner diameter of $15.9 \mathrm{~mm}$, an outer diameter of $40.0 \mathrm{~mm}$ and a depth of $3.3 \mathrm{~mm}$. The plasma sheath edge partially conforms to the groove, creating an annular depression in the equipotential surfaces above the groove. The dust particles are illuminated using a steady sheet of 635-nm laser light. Particle positions are recorded using a video camera. For these experiments 1025 frames of $1024 \times 1024$ pixels were recorded at 7.51 frames/s.

Making a uniform dusty plasma ring [Fig. 2] requires the bottom of the potential well to be flat. This condition is more stringent than confinement in a parabolic well since a tilted parabolic well is still parabolic. To create a dust ring we adjusted the tilt of the electrode 


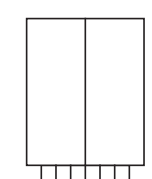

$1 / 2$ in. CCD

video camera

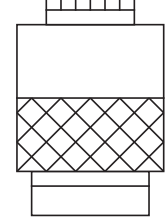

$0.75 x$ adapter

$55 \mathrm{~mm} \mathrm{f} / 2.8$

telecentric lens

red filter

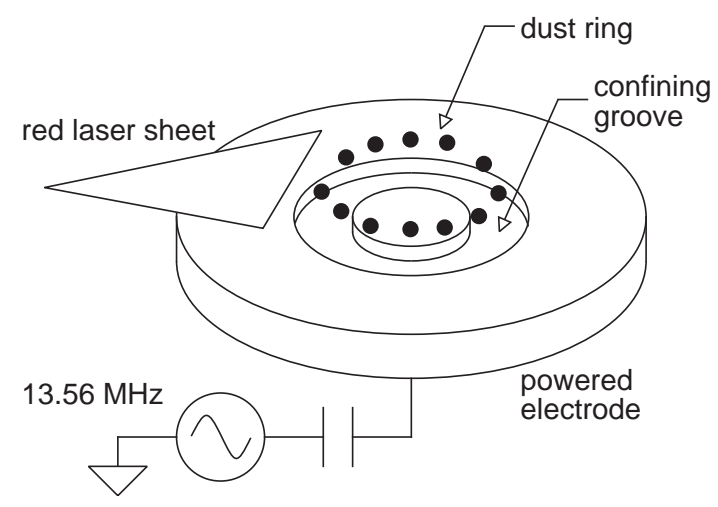

Figure 1: Schematic of the experimental setup. An annular groove with rectangular cross section cut into the rf electrode produces an annular confining well for dust particles. When the particle spacing is above a critical value the particle configuration is one-dimensional with a ring topology.

(actually the entire vacuum vessel) until the particles were distributed approximately uniformly in a ring. However, at low pressures $(\lesssim 20$ mtorr $)$ we observed an $m=2$ azimuthal perturbation to the potential well. The exact characteristics of this perturbation depend on the rf power, suggesting it may be due to an rf standing-wave component. Such a perturbation cannot be eliminated by tilting the electrode. We used two grounded L-shaped rods inserted horizontally above the electrode to cancel this perturbation by varying the distance and orientation of the rods.

An equilibrium configuration of a dusty plasma 1-ring is shown in Fig. 2(a). The ring consists of $n=68$ dust particles floating $9.2 \mathrm{~mm}$ above the top of the electrode. Discharge parameters were a neutral pressure of 18.9 mtorr argon, $\sim 3 \mathrm{~W}$ forward rf power at 13.56 $\mathrm{MHz}$, and the dc self-bias on the electrode was $-52 \mathrm{~V}$. Wave modes are underdamped at this pressure. The camera resolution was $0.0317 \mathrm{~mm} /$ pixel . To determine the geometric center of the ring we fitted the particle coordinates to a circle [22] and then averaged over all frames. (Because of variable particle spacing the geometrical center and the center of 

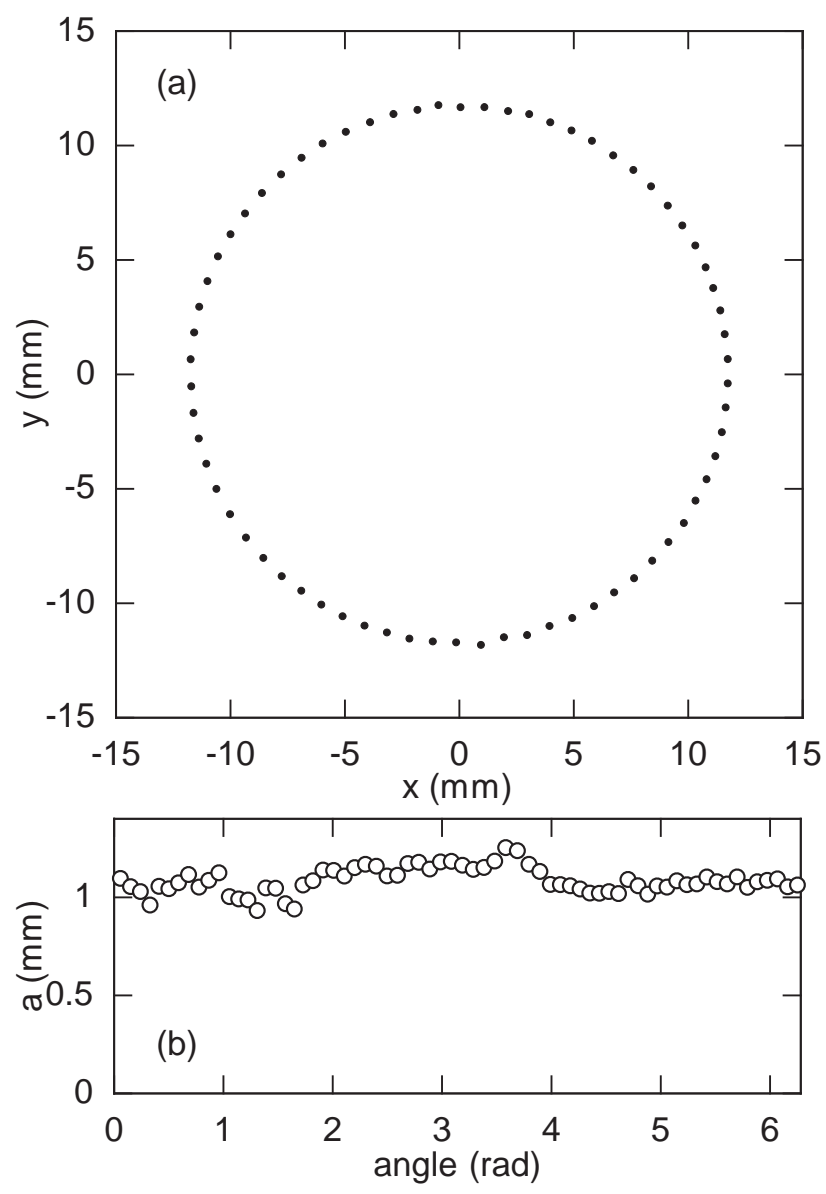

Figure 2: (a) Measured equilibrium positions of $n=68$ particles confined in a two-dimensional annular well. The particles form a one-dimensional ring. (b) Distance $a$ between nearest neighbors vs azimuthal angle.

mass are not identical.) We found an average radius $R=11.73 \mathrm{~mm}$, so that the average particle separation computed as the circumference over $n$ is $a=1.084 \mathrm{~mm}$. The dust ring lies closer to the inner edge of the groove. The Euclidean distance $r_{i j}$ between adjacent pairs of particles is shown in Fig. 2(b). The average separation $\left\langle r_{i j}\right\rangle=1.084 \pm 0.008 \mathrm{~mm}$ agrees with the previous estimate of $a$.

In Fig. 3(a) we demonstrate that it is also possible using this confinement geometry to create a quasi-1D configuration of two rings (i.e., a 2-ring) with different radii, as predicted by theory [13, 14]. In this case, we have a total of $n=87$ particles, with 43 particles in the inner ring and 44 particles in the outer ring. The radius of the inner ring is $10.12 \pm 0.01$ $\mathrm{mm}$, and that of the outer ring is $11.24 \pm 0.01 \mathrm{~mm}$. For most of this two-ring the particles are in a zigzag configuration. However, near $0^{\circ}$ the two rings "slip" past each other. The 

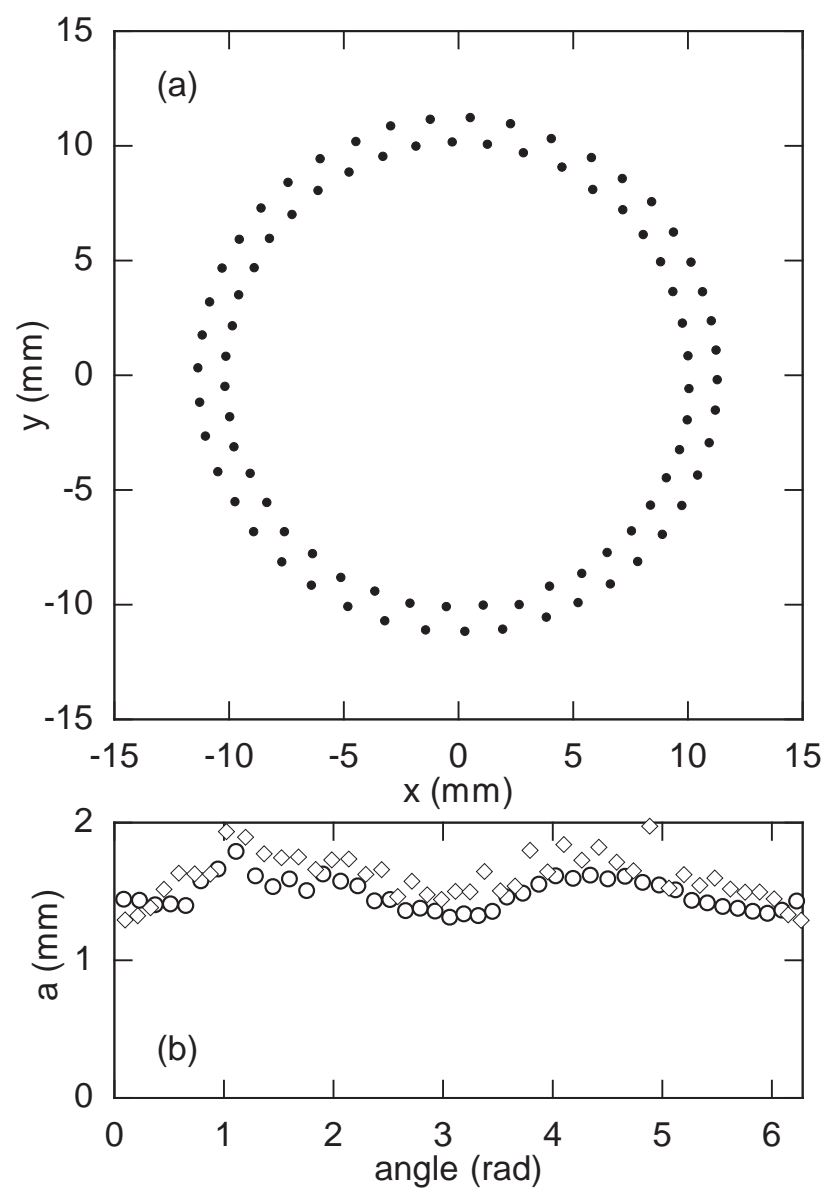

Figure 3: (a) Quasi-one-dimensional configuration (a 2-ring) consisting of $n=87$ particles arranged in two concentric rings with 43 particles in the inner ring and 44 in the outer ring. (b) Distance between particles in the inner ring (open circles) and in the outer ring (open diamonds) vs angle.

distance between pairs of particles in the inner and outer rings is plotted in Fig. 3(b). The average distance between particles in the inner ring is $1.48 \pm 0.02 \mathrm{~mm}$, and $1.60 \pm 0.03 \mathrm{~mm}$ for the outer ring. An $m=2$ modulation in the particle spacing is visible.

The particle positions undergo small amplitude thermal fluctuations, mostly due to their interaction with the neutral gas component [18]. For the 1-ring [Fig. 2(a)], velocity distributions in the longitudinal (azimuthal) and transverse (radial) directions were computed from the time histories of the measured particle positions [23]. The velocity distribution functions indicate a minimum measurable velocity of $\approx 0.06 \mathrm{~mm} / \mathrm{s}$, where a velocity of 1 pixel/frame corresponds to $v=0.238 \mathrm{~mm} / \mathrm{s}$. Except for a small neighborhood around $v=0$, the distributions in both directions are well fitted by a Maxwellian with a temperature $T=410 \mathrm{~K}$, somewhat below $530 \mathrm{~K}$ reported for a $2 \mathrm{D}$ lattice [23]. This temperature indicates that the 
ring is stable and in equilibrium with the neutral gas.

The dependence of the power spectral density for the thermal fluctuations vs wavenumber $k$ for the 1-ring were computed for the longitudinal (acoustic) and transverse (optical) modes. First, the average position of each particle was computed by averaging over all 1025 frames. Then, for each frame the longitudinal and transverse displacements were computed and Fourier analyzed using a discrete Fourier transform (DFT) to give the amplitude and phase of each $k$ vs time, which assumes the equilibrium lattice spacing is independent of position. The power spectral density (psd) of the time history of each wavenumber was then computed using the maximum entropy method (MEM) [24]. The psd's from four data sets were averaged to give the final psd. Since each normal mode acts as an independent damped harmonic oscillator, the psd peaks near the mode's natural frequency and so the maximum of the psd should follow the dispersion relations [11].

The power spectral density for the acoustic modes is displayed in Fig. 4(a). Here the horizontal axis runs from mode number 0 to 34 , or equivalently $k a=0$ to $\pi$. The vertical axis starts at $0.5 \mathrm{~Hz}$ because of low-frequency noise and runs to the Nyquist frequency of 3.75 Hz. The power spectral density for the transverse mode is shown in Fig. 4(b). Here we see a typical optical mode, with a finite frequency for $k=0$, which should be $\omega_{0}$ [Eq. (2)], and then a decrease in $\omega$ with $k$ indicating a backwards wave. The $m=1$ mode displays several narrow band features due to minute mechanical vibrations coupled to the vacuum chamber from our belt-drive mechanical pump. In this case, the electrode and camera oscillate while the dust ring remains stationary.

We fitted the psd for each mode with the equation for a driven damped harmonic oscillator for both the longitudinal and transverse directions to determine the natural mode frequency $\omega_{i}$ vs $k$. Here $\omega_{i}$ may be slightly above the maximum of the psd because of finite damping, where the damping rate $\gamma \sim 2 \mathrm{~s}^{-1}$. We then fitted both measured dispersion relations simultaneously to theory [Eqs. (8) and (9)], where there are only three free parameters: $\omega_{0}, \alpha$ and $\bar{\kappa}$. From this procedure, we found $\omega_{0}=16.6 \mathrm{rad} / \mathrm{s}, \alpha=1.41$ and $\bar{\kappa}=1.32 \pm$ 0.05. We believe that this is the first report of very good agreement of experimental data simultaneously with both branches of the dispersion relation, confirming that this system is well described by a model for an unbounded 1D chain of particles interacting through a Yukawa potential. For $\bar{\kappa}=1.32$ the critical value of the lattice parameter $\alpha=a / r_{0}$ for the zigzag instability is $\alpha_{c}=1.13$. For the ring studied here, $\alpha=1.41$ is significantly above 


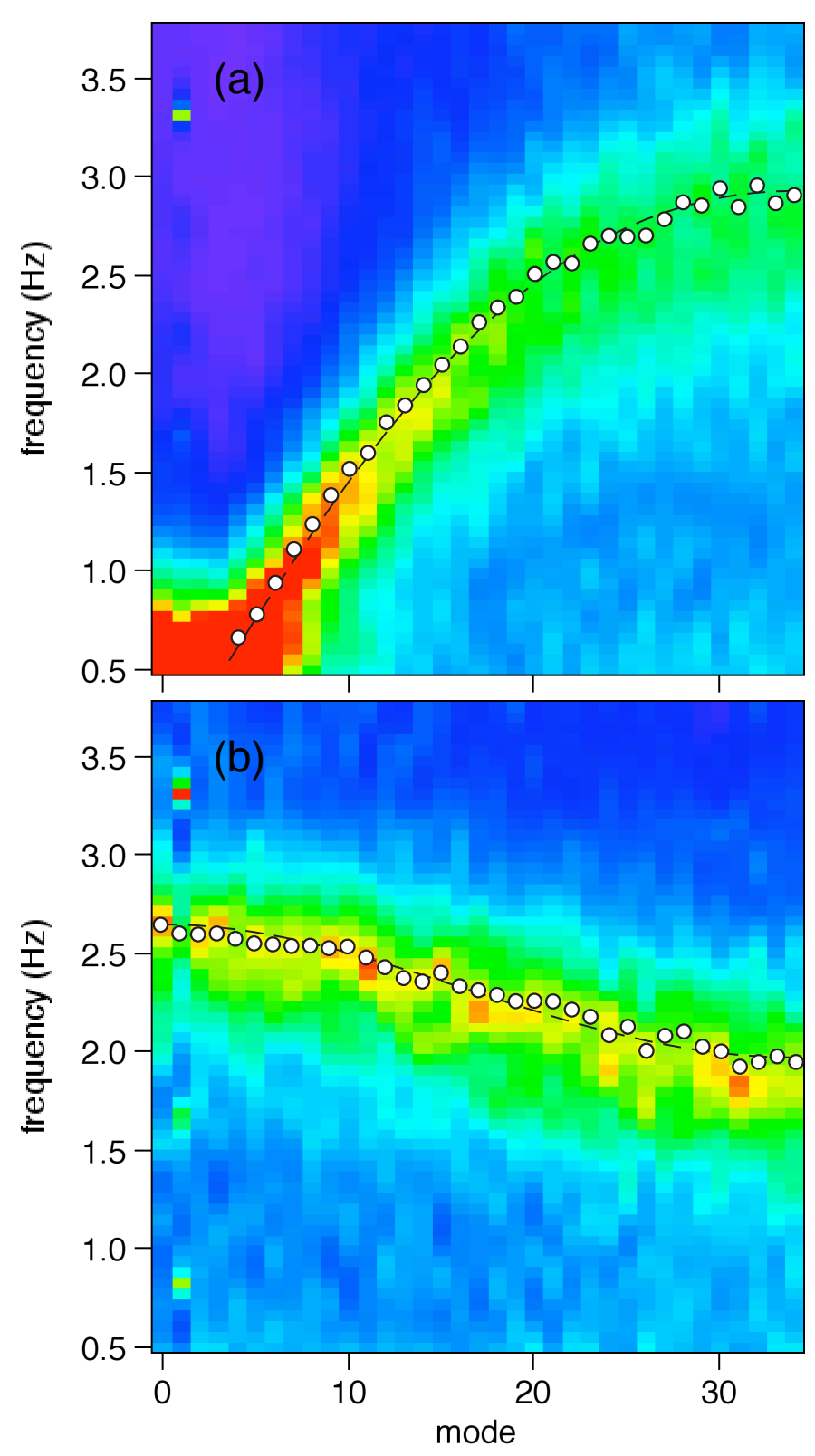

Figure 4: (Color online) (a) Power spectral density (psd) for thermally-excited longitudinal modes vs mode number and frequency and (b) psd for transverse modes. Purple corresponds to the least, and red to the greatest, power density. Open circles are the natural frequency for each mode, and the dashed line is the fit to the theoretical dispersion relations.

$\alpha_{c}$. Dimensional parameters are $r_{0}=0.77 \mathrm{~mm}, \lambda_{D}=0.82 \mathrm{~mm}$ and $q=-1.24 \times 10^{4} e$. The values of $\lambda_{D}$ and $q$ are consistent with those found in DONUT for similar plasma conditions [7. 18,21$]$.

In summary, we have created $1 \mathrm{D}$ and quasi-1D dusty plasma rings experimentally, verify- 
ing previous predictions for charged particles confined in a 2D annular well [13, 14]. Measured longitudinal and transverse dispersion relations in the 1-ring exhibit excellent agreement with dispersion relations in an unbounded Yukawa lattice, confirming that these systems are well described by a model of $n$ identical charged particles confined in an annular potential and interacting through a Yukawa potential. These rings provide a new experimental system for studying static and dynamic properties of 1D and quasi-1D systems.

[1] N. W. Ashcroft and N. D. Mermin, Solid State Physics (W. B. Saunders, Fort Worth, 1976), pp. $430-3$.

[2] E. Fermi, J. R. Pasta and S. Ulam, Studies of Nonlinear Problems I, Report LA-1940 (Los Alamos Scientific Laboratory, Los Alamos, 1955).

[3] M. Toda, Nonlinear Waves and Solitons (KTK Scientific Publishers, Tokyo, 1989).

[4] U. Konopka, G. E. Morfill and L. Ratke, Phys. Rev. Lett. 84, 891 (2000).

[5] M. Lampe, G. Joyce, G. Ganguli and V. Gavrishchaka, Phys. Plasmas 7, 3851 (2000).

[6] T. E. Sheridan, Phys. Plasmas 14, 032108 (2007).

[7] T. E. Sheridan and K. D. Wells, Phys. Rev. E 81, 016404 (2010).

[8] A. Homann, A. Melzer, S. Peters and A. Piel, Phys. Rev. E 56, 7138 (1997).

[9] T. Misawa, N. Ohno, K. Asano, M. Sawai, S. Takamura and P. K. Kaw, Phys. Rev. Lett. 86, 1219 (2001).

[10] B. Liu, K. Avinash and J. Goree, Phys. Rev. Lett 91, 255003 (2003).

[11] B. Liu and J. Goree, Phys. Rev. E 71, 046410 (2005).

[12] A. Melzer, Phys. Rev. E 73, 056404 (2006).

[13] I. V. Schweigert, V. A. Schweigert and F. M. Peeters, Phys. Rev. B 54, 10827 (1996).

[14] T. E. Sheridan, Phys. Scr. 80, 065502 (2009).

[15] F. Melands $\varnothing$, Phys. Plasmas 3, 3890 (1996).

[16] K. Nelissen, V. R. Misko and F. M. Peeters, EPL 80, 56004 (2007).

[17] G. Piacente, I. V. Schweigert, J. J. Betouras and F. M. Peeters, Phys. Rev. B 69, 045324 (2004).

[18] T. E. Sheridan and W. L. Theisen, Phys. Plasmas 13, 062110 (2006).

[19] T. E. Sheridan, Phys. Rev. E 72, 026405 (2005). 
[20] T. E. Sheridan, J. Appl. Phys. 106, 033303 (2009).

[21] T. E. Sheridan, K. D. Wells, M. J. Garee and A. C. Herrick, J. Appl. Phys. 101, 113309 (2007).

[22] A. Al-Sharadqah and N. Chernov, Electronic Journal of Statistics 3, 886 (2009).

[23] S. Nunomura, J. Goree, S. Hu, X. Wang, A. Bhattacharjee and K. Avinash, Phys. Rev. Lett. 89, 035001 (2002).

[24] W. H. Press, S. A. Teukolsky, W. T. Vetterling and B. P. Flannery, Numerical Recipes in C, 2nd ed. (Cambridge University Press, Cambridge, 1992), pp. 572-5. 